\title{
Prevalência da Hipertensão Arterial Sistêmica na População Urbana de Passo Fundo (RS)
}

\author{
Ibsen S. Trindade, Gilberto Heineck, Josemar Roberto Machado, Henrique Ayzemberg, Maurice \\ Formighieri, Mosara Crestani, Jefferson Gusso
}

Passo Fundo, RS

\begin{abstract}
Objetivo - Avaliar a prevalência de hipertensão arterial sistêmica (HAS) na população urbana da cidade de Passo Fundo (RS).

Métodos - Estudo observacional, descritivo e transversal de base populacional de uma amostra aleatória significativa da população em estudo. O critério para HASfoi 160/95mmHg. A média das últimas três aferições da pressão arterial (PA) foi utilizada nas análises. A amostra foi composta por 206 indivíduos. Houve correção dos níveis pressóricos em relação ao diâmetro do braço. A entrevista foi feita com questionários estandardizados.

Resultados - A prevalência da HAS foi de 21,9\% $(I C=19,3$ a 24,5) utilizando o critério de 160/95 somado aos pacientes normotensos em uso regular de medicação anti-hipertensiva. Dos 45 individuos hipertensos, 53,3\% tomavam medicação anti-hipertensiva regularmente, sendo que $20 \%$ estavam com a PA controlada. Da população em estudo, 4,4\% eram diabéticos, 33,0\% fumantes, 31,4\% mulheres usando anticoncepcional oral, 2,9\% abusando de álcool, 29,6\% obesos. Encontramos associação significativa com a HAS, em relação à idade, obesidade e diabetes mellitus e não a encontramos com referência ao sexo, cor, abuso de álcool, fumo e uso de anticoncepcional oral.

Conclusão - A prevalência da HAS em Passo Fundo está dentro dos limites esperados para tal; no entanto, o grau de controle desta população está em um nível muito aquém do satisfatório.
\end{abstract}

Palavras-chave: hipertensão arterial sistêmica, epidemiologia

\section{Prevalence of Hypertension in the Passo Fundo (Brazil) Metropolitan Area}

Purpose - To evaluate the present prevalence of hypertension in the Passo Fundo metropolitan area.

Methods - This is an observational, descriptive, crossectional design with populational base, of a representative random sample of the adults of this urban region. The criteria for hypertension were blood pressure $\geq 160 / 95 \mathrm{mmHg}$ or antihypertensive treatment. The average of the last three measurements was used in the analysis. Two hundred and six subjects were selected. The interview was made with standardized questionnaires. Blood pressure was corrected for arm diameter.

Results - The prevalence of hypertension was $21.9 \%$ $(C I=19.3$ to 24.5$)$ of 45 hypertensive subjects, $53.3 \%$ were using anti-hypertensive drugs regularly, however only $20 \%$ had normal BP levels. Among the study population $4.4 \%$ were diabetics, $33.0 \%$ were smokers, $31.4 \%$ of the women were using contraceptives, $2.9 \%$ were heavy alcohol users, $29.6 \%$ were obese. The following conditions of interest were significatively associated with hypertension: age, obesity and diabetes; there was no association with sex, color, heavy alcohol use, smoking and contraceptive use.

Conclusion - The prevalence of hypertension in the Passo Fundo metropolitan area is within the expected range, however the level of control in this population is not acceptable.

Key-words: hypertension, epidemiology

Arq Bras Cardiol, volume $71\left(n^{\circ} 2\right), 127-130,1998$

As doenças cardiovasculares são um importante problema de saúde pública e a principal causa de morte da população adulta dos países desenvolvidos.

Faculdade de Medicina da Universidade de Passo Fundo

Correspondência: Ibsen S. Trindade - Rua Teixeira Soares, 777/401 - 99010-081

- Passo Fundo, RS

Recebido para publicação em 6/10/97

Aceito em 3/6/98
Desde há muito, já se tem o conhecimento baseado em evidências clínicas da importância da hipertensão arterial sistêmica (HAS), como fator de risco na morbimortalidade na doença isquêmica cardiovascular.

Apesar do imenso arsenal médico para o tratamento desta doença, nem todos têm acesso a ele e, em muitos casos, o diagnóstico é feito tardiamente ou, às vezes, nem mesmoé feito. 
O Brasil está sofrendo uma transição epidemiológica, que significa a mudança na incidência das causas de mortalidade, passando de causas infecto-contagiosas e maternas, as mais incidentes, para doenças crônico degenerativas, como a HAS.

Assim sendo, torna-se necessária uma avaliação correta da prevalência da HAS na população geral de nossa cidade, pois isto tem implicações epidemiológicas, sociais e políticas, sendo que estes dados serão úteis para a planificação das ações de saúde preventiva, assistencial e terapêutica desta população.

O presente trabalho tem como objetivo determinar a prevalência da HAS na população adulta da região urbana da cidade de Passo Fundo e apresenta alguns aspectos populacionais relacionados a esta doença.

\section{Métodos}

Este trabalho é um estudo observacional, descritivo e transversal de base populacional. Foram realizadas entrevistas domiciliares ou no local de trabalho, em moradores da zona urbana da cidade de Passo Fundo, com idades entre 18 e 74 anos. As entrevistas foram realizadas de junho a agosto de 1995.0 número da amostra foi calculado previamente com base em uma população de 87.593 indivíduos que residiam na zona urbana da cidade e estavam dentro da faixa etária estipulada (IBGE, 1991) com uma precisão de estimativa de prevalência de HAS de $\pm 5 \%$ para um intervalo de confiança de $95 \%$.

Para determinar a pessoa a ser entrevistada, utilizou-se o mapa do perímetro urbano de Passo Fundo de 1993, ofertado pela Prefeitura Municipal, o mais recente na ocasião da execução do trabalho. Todas as quadras da cidade foram numeradas uma a uma (2259 quadras). Como o número era de 206 indivíduos, foram sorteadas 206 quadras; em cada quadra sorteada selecionado um indivíduo da amostra. Para chegar a um indivíduo específico, foi usada uma tabela de números aleatórios, pela qual se estabelecia um lado da quadra e após, a casa ou apartamento a ser visitado. $\mathrm{O}$ indivíduo escolhido, nesta casa ou apartamento, era o $1^{\circ}$ aniversariante a partir da data da entrevista. Em caso de recusa, ou o indivíduo não ser encontrado após três visitas, ele era excluído da amostra e em seu lugar era incluída a primeira residência à esquerda, sendo o indivíduo determinado do mesmo modo. Em quatro quadras sorteadas não havia habitações, sendo então selecionadas novas quadras.

A entrevista, bem como as medidas da pressão arterial (PA), foram executadas pelos autores. O questionário foi avaliado previamente em um estudo piloto, sendo corrigidas as falhas na compreensão das perguntas pela população.

Para a determinação da PA, foram utilizados esfigmomanômetros anaeróides de várias marcas, previamente calibrados no serviço de Engenharia Biomédica do Hospital São Vicente de Paulo de Passo Fundo, segundo um sistema integrado para calibração de esfigmomanômetros ${ }^{1}$.

Os pesquisadores trabalharam em duplas; um era o entrevistador e o outro realizava a medida da PA e a medida do diâmetro do braço. A técnica da medida pressórica seguida foi a recomendada por Kaplan ${ }^{2}$, havendo correção para o perímetro braquial segundo tabela de Mion e $\mathrm{col}^{3 .}$

Os entrevistadores foram considerados aptos a ir a campo quando, após a discussão do método a ser seguido e um período de treinamento, a medida da PA realizada pelo entrevistador correspondia à medida do investigador principal do trabalho, medidas estas realizadas em pacientes internados no hospital escola da Faculdade de Medicina da Universidade de Passo Fundo.

Foram realizadas quatro aferições da PA (uma no início da entrevista, duas durante e uma após) com intervalo mínimo de 5 min entre elas. Para fins de análise, considerouse a média das últimas três medidas da PA desde que não houvesse uma diferença maior que $5 \mathrm{mmHg}$ entre elas (a $1^{\mathrm{a}}$ medida foi sempre descartada).

Foi definido como hipertenso o indivíduo que apresentava uma pressão sistólica $>160 \mathrm{mmHg}$ e/ou uma diastólica $\geq 95 \mathrm{mmHg}{ }^{4,5}$, ou o indivíduo sabidamente hipertenso, que vinha em uso regular de medicação anti-hipertensiva, e que podia estar com níveis pressóricos elevados ou não no momento da entrevista.

Foi definido como fumante, aquele que relatava fumar pelo menos um cigarro por dia e ex-fumante, aquele que fumava regularmente no passado e que abandonou por completo o tabagismo ${ }^{6}$.

Em relação ao consumo de álcool, foram feitas questões sobre frequiência, tipo e quantidade de bebida ingerida, que permitiram o cálculo de gramas de álcool ingeridas por semana. Foi considerado consumidor excessivo de álcool os homens que ingeriam quantidade $\geq 350 \mathrm{~g}$ de álcool por semana e as mulheres quantidade $\geq 210 \mathrm{~g}$ de álcool no mesmo período ${ }^{7}$.

Os entrevistados foram indagados sobre os valores do seu peso e altura; levando-se em conta que essas medidas tivessem excelente relação com as medidas reais $(\mathrm{r}=97 \%)^{8}$, calculou-se com esses dados o índice de massa corporal $(\mathrm{IMC}=$ peso em $\mathrm{kg}$ dividido pelo quadrado da altura em metro). O ponto de corte acima do qual considerou-se obesidade, foi de $27 \mathrm{~kg} / \mathrm{m}^{2}$.

Para a análise do uso de anticoncepcional oral (ACO) na HAS, somente foram levadas em conta as mulheres em idade fértil.

Para os cálculos estatísticos das variáveis categóricas utilizou-se o qui-quadrado com correção de Yates ou o teste exato de Fisher quando necessário. O nível de significância estatística adotado foi de 0,05 .

\section{Resultados}

Na amostra estudada, $58,7 \%$ pertenciam ao sexo feminino e $41,3 \%$ ao masculino, tornando-se a amostra representativa da população em estudo.

A idade média foi de 41,7 $\pm 13,9$ anos, sendo que no grupo dos homens, a idade média foi maior $(44,28 \pm 14,77 \mathrm{vs}$ $39,9 \pm 13,05)$.

Quanto à raça, determinada pelo entrevistador, 92,7\% 


\begin{tabular}{|c|c|c|c|c|}
\hline Variável & Condição & $\begin{array}{l}\text { HAS sim } \\
(\mathrm{N}=45)\end{array}$ & $\begin{array}{l}\text { HAS não } \\
(\mathrm{N}=161)\end{array}$ & $\mathrm{p}$ \\
\hline \multirow[t]{2}{*}{ Sexo } & Masculino & $21(24,7)$ & $64(74,3)$ & \multirow[t]{2}{*}{0,508} \\
\hline & Feminino & $24(19,8)$ & $97(80,2)$ & \\
\hline \multirow[t]{2}{*}{ IMC } & $>27$ & $22(36,1)$ & $39(63,9)$ & \multirow[t]{2}{*}{0,002} \\
\hline & $<27$ & $23(15,9)$ & $122(84,1)$ & \\
\hline \multirow[t]{2}{*}{ Diabetes mellitus } & Sim & $5(55,6)$ & $4(44,4)$ & \multirow[t]{2}{*}{0,025} \\
\hline & Não & $40(20,3)$ & $157(79,7)$ & \\
\hline \multirow[t]{6}{*}{ Idade } & $18-27$ & 0 & $28(100)$ & \multirow[t]{6}{*}{$<0,001$} \\
\hline & $28-37$ & $7(10,6)$ & $59(89,4)$ & \\
\hline & $38-47$ & $9(18)$ & $41(82)$ & \\
\hline & $48-57$ & $10(38,5)$ & $16(61,5)$ & \\
\hline & $58-67$ & $14(56)$ & 11 (44) & \\
\hline & $>68$ & $5(45,5)$ & $6(54,5)$ & \\
\hline \multirow[t]{2}{*}{ Fumo } & Sim & $13(19,1)$ & $55(80,9)$ & \multirow[t]{2}{*}{0,627} \\
\hline & Não & $32(23,2)$ & $106(76,8)$ & \\
\hline \multirow[t]{2}{*}{ Abuso de álcool } & Sim & $2(33,3)$ & $4(66,7)$ & \multirow[t]{2}{*}{0,390} \\
\hline & Não & $43(21,5)$ & $157(78,5)$ & \\
\hline \multirow[t]{2}{*}{ Cor } & Branco & $40(20,9)$ & $151(70,1)$ & \multirow[t]{2}{*}{0,427} \\
\hline & Não branco & $5(33,3)$ & $10(66,7)$ & \\
\hline \multirow[t]{2}{*}{ Anticoncepcional } & Uso & $3(7,9)$ & $35(92,1)$ & \multirow[t]{2}{*}{1.00} \\
\hline & Não Uso & $4(8,2)$ & $45(91,8)$ & \\
\hline
\end{tabular}

eram brancos, $4,4 \%$ eram pretos, $2,4 \%$ amarelos e $0,5 \%$ foram classificados como outros.

A pergunta inicial era se o paciente tinha conhecimento de ser portador ou não de HAS; $42(20,4 \%)$ responderam que sim, $117(56,8 \%)$ afirmavam que não eram hipertensos e $47(22,8 \%)$ não tinham conhecimento.

Dos 42 entrevistados que se diziam hipertensos, 32 (76,2\%) faziam algum tipo de tratamento, sendo que, 29 faziam uso de medicação, e três não. Dos 29 entrevistados que tomavam remédios, $24(82,8 \%)$ diziam seguir a prescrição médica fielmente e $5(17,2 \%)$ não. Os três entrevistados que não tomavam medicação e que diziam se tratar, estavam fazendo dieta com restrição salina e um também realizava exercícios físicos com o objetivo de reduzir os níveis pressóricos. Dos outros 10 indivíduos, que se diziam hipertensos, mas que não faziam tratamento, identificamos cinco hipertensos e cinco não hipertensos.

Da amostra em estudo, $9(4,4 \%)$ relatavam ser diabéticos, $171(83,0 \%)$ sabiam não ser diabéticos e 26 (12,6\%) não sabiam informar.

Quanto ao tabagismo, 68 (33,0\%) foram considerados fumantes, segundo os nossos critérios, sendo que, 32 $(47,1 \%)$ fumavam até 10 cigarros ao dia, $31(45,6 \%)$ de 11 a 20 cigarros ao dia e $5(7,3 \%)$ fumavam acima de 20 cigarros ao dia. Dos 138 restantes que não eram fumantes, $38(27,5 \%)$ já haviam fumado no passado.

Das 121 mulheres, $38(31,4 \%)$ estavam emidade fértile em uso de anticoncepcional oral.

Quanto ao consumo de álcool, 131 (63,6\%) tomavam regularmente bebida alcoólica, porém consumo excessivo de álcool só encontramos em 6 (2,9\%) homens entrevistados.
A amostra em estudo apresentou um peso médio de $68,3 \pm 13,5 \mathrm{~kg}$ e uma altura média de $164,2 \pm 7,9 \mathrm{~cm}$. Quanto ao IMC $\geq 27$ (critério de obesidade), encontramos em 61 $(29,6 \%)$ indivíduos.

A prevalência de HAS foi de 21,9\% (45 indivíduos considerados hipertensos de uma amostra de 206) pelo critério de 160/95mmHg ou normotenso em uso regular de medicação anti-hipertensiva. Dos 45 indivíduos hipertensos, 37 já conheciam a sua situação e oito não.

Realizando a análise da prevalência da HAS, em relação às condições de interesse (tab. I), não se encontrou associação significativa com sexo ( $\mathrm{p}=0,508)$, cor $(\mathrm{p}=0,427)$, abuso de álcool $(\mathrm{p}=0,390)$, fumo $(\mathrm{p}=0,627)$ e uso de ACO $(\mathrm{p}=1,000)$, mas, sim com a idade $(\mathrm{p}<0,001)$, obesidade $(\mathrm{p}=$ $0,002)$ e diabetes mellitus $(\mathrm{p}=0,025)$.

\section{Discussão}

Quando comparados os nossos resultados com os trabalhos já existentes da prevalência da HAS no Rio Grande do Sul ${ }^{5-9}$, levando em conta pacientes hipertensos, e normotensos com PA controlada em uso regular de medicação anti-hipertensiva, identificou-se um valor um pouco maior. Achutti e Medeiros encontraram 17,4\% de hipertensos e, mais recentemente, Fucks e col encontraram $19,5 \%$; o nosso resultado de $21,9 \%$, apesar de ser maior, não difere estatisticamente do de Fucks ( $\mathrm{p}=0,5$; IC=95\%). Quando excluídos os pacientes com HAS em tratamento regulare com PA controlada, Achutti encontrou 11,8\%, Fucks e col $12,6 \%$ e nós $17,5 \%$. Novamente, apesar de ser maior a nossa prevalência, não há diferença estatística com os resultados de Fucks e col ( $p>0,05$, IC=95\%).

Dos parâmetros analisados, encontrou-se associação positiva com a HAS, a idade, a obesidade, o diabetes mellitus, não se encontrando associação com o sexo, cor, consumo abusivo de álcool, uso de tabaco e nem com o uso de anticoncepcional oral. Acreditamos que a falta de associação com a variável consumo abusivo de álcool foi devido a um baixo índice desses indivíduos identificados em nosso meio (apenas 2,9\%), provavelmente conseqüente à inibição de fornecer a informação por parte dos entrevistados. A não associação com a variável cor, também observada por Fucks e col, é devido ao baixo índice de negros em nossa cidade $(4,4 \%)$ cuja colonizaçãoé predominantemente de alemães e de italianos. Não se encontrou relação direta da HAS com a variável sexo, também não encontrada por vários autores, porém não subdividimos os sexos por idade, acima e abaixo dos 55 anos, onde supostamente haveria alguma diferença. A não observação de HAS entre os fumantes, no nosso trabalho, decorre da observação que o efeito hipertensivo do fumo dura somente até 15 a 30 min após o último cigarro, o que nos faz acreditar que medimos a PA desta população específica após este tempo - os pacientes foram orientados a não fumar durante a entrevista, porém não foi relatado se haviam fumado recentemente ou não. No entanto, vem de encontro aos nossos resultados, o fato de que a associação entre HAS e fumo só foi bem determinada com o advento e 
a utilização da monitorização ambulatorial da pressão arterial (MAPA) ${ }^{10}$, método não utilizado no nosso trabalho. Quanto ao anticoncepcional oral, é sabido que a HAS aparecerá em $5 \%$ das mulheres que usam anticoncepcional oral por, no mínimo, cinco anos ${ }^{11}$. No entanto, o número de mulheres hipertensas em uso de anticoncepcional foi pequeno (apenas três) e não se levou em conta o tempo de uso, e nem o tipo de ACO, pois a quantidade de estrogênio e progesterona dos ACO mais modernos são muito menores que os mais antigos determinando uma menor associação com problemas cardiovasculares.

A proporção de hipertensos que sabia de sua condição era de $82,2 \%$, proporção esta, alta em relação aos $57,7 \%$ encontrados por Fucks e col. Em relação ao tratamento medicamentoso, observamos os seguintes dados: a) dos 45 hipertensos identificados, $24(53,3 \%)$ estavam em tratamento medicamentoso regular; sendo que apenas $9(37,5 \%)$ deles estavam com os níveis pressóricos controlados. Assim, considerando-se toda a população de hipertensos (45 indivíduos), observa-se que apenas $20 \%$ estavam em tratamento regular e com PA controlada.

Os pacientes que se diziam hipertensos e que em nossos critérios não haviam sido classificados como tal (cinco indivíduos), demonstraram a existência de falsos hipertensos entre a população, os quais haviam sido previamente rotulados como hipertensos por provável(eis) erro(s) no processo de medição e avaliação da PA a que haviam sido submetidos.

Detectaram-se $8(17,8 \%)$ casos de hipertensão entre a população estudada, que até o momento desconhecia essa situação, representando a evidência de um segmento da população que sendo hipertenso não tinham conhecimento da doença.

Uma das limitações do trabalho é que a medida foi feita em uma só visita domiciliar, determinando uma superestimação na avaliação da prevalência da HAS.

Assim, concluiu-se que a prevalência de hipertensos na população urbana da cidade de Passo Fundo é de 21,9\%, sendo que este dado vem de encontro aos trabalhos regionais já publicados e à literatura médica mundial. O perfil da nossa população de hipertensos, em relação às condições de interesse estudadas, é similar aos grupos já estudados de nosso estado. Frente aos resultados obtidos, e considerando que há uma população de hipertensos não identificados, supõe-se que há entre a população estudada, aproximadamente, 3.414 indivíduos com HAS e sem diagnóstico, que devem ser identificados e tratados. Os nossos hipertensos possuem um grau de controle inferior ao já relatado na literatura, o que nos permite indagar sobre a eficiência de nosso sistema de saúde. A necessidade não é tão somente em identificar os casos de hipertensão, mas também, em melhorar o acesso e a observância ao tratamento.

\section{Referências}

1. Spalding LES - Sistema integrado de instrumentos e procedimentos para a realização de calibração de esfigmomanômetros (Dissertação para a obtenção do grau de mestre). Florianópolis, 1995.

2. Kaplan NM - Measurement of Blood Pressure. In: Kaplan NM, eds. Clin

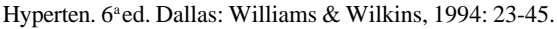

3. Mion Jr.D, Silva HB, Marcondes M - Novo dispositivo para correção de leitura da pressão arterial de acordo com a circunferência do braço do paciente. Anais XII Congresso Brasileiro de Nefrologia, Salvador, Bahia 1984.

4. The Fifth Report of Joint National Committee on Detection, Evaluation, and Treatment of High Blood Pressure. Arch Intern Med 1993; 153: 154-83.

5. Fuchs FD, Moreira LB, Moraes RS, Bredemeier M, Cardozo SC - Prevalência da hipertensão arterial sistêmica e fatores associados na região urbana de Porto Alegre. Estudo de base populacional. Arq Bras Cardiol 1994, 63: 473-9.

6. World Health Organization - Guidelines for the conduct of tobacco smoking surveys of the general population -Report of a WHO meeting held in Helsinki, Finland 1982. Geneva, 1983. Publication WHO/SMO/83.4.

7. Wallace P, Cultler S, Haines A - Randomized controlled trial of general practioner intervention in patients with excessive alcohol consumption. $\mathrm{Br} \mathrm{Med}$
J 1988; 297: 663-8

8. Zimmer PM, Pellanda LC, Tavares MRG, Duncan BB, Schmidt MI - Validade do peso referido para uso em estudos epidemiológicos - In: $7^{\circ}$ Congresso Brasileiro de Diabetes, Guarapari, 1989. Programa Científico: 48.

9. Achutti A, Medeiros AMB - Hipertensão arterial no Rio Grande do Sul. Porto Alegre: B. Saúde da SSMA - RS 1985; 12: 2-72.

10. Mann SJ, James GD, Wang RS, Pickering TG - Elevation of ambulatory systolic blood pressure in hypertensive smokers. A case control study. JAMA 1991; 265: 2226-8.

11. Kaplan NM - Clinical Hypertension. 6 ${ }^{\mathrm{a}}$ ed. Dallas: Williams \& Wilkins 1994: 358.

12. Beltrame AB - Hypertension in Latin America: Importance and approaches to control. Clin Exper Hyperten 1993; 15: 1005-13.

13. Ruotolo SL, Rinaldi GJ, Almiron MA, Gende OA, Cingolani - Consideraciones sobre la definicion de hipertension arterial a partir de un estudio de prevalencia en 1423 jovenes - Medicina (Buenos Aires) 1992; 52: 119-30.

14. II Consenso Brasileiro para o Tratamento da Hipertensão Arterial. Arq Bras Cardiol 1994; 63: 333-47. 